\title{
Three-Dimensional Time Domain Simulation of the Quantum Magnetic Susceptibility
}

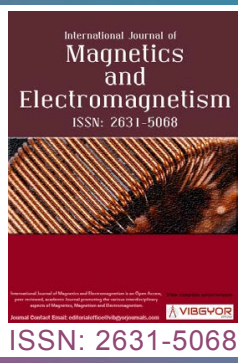

Jennifer Houle ${ }^{1}$, Dennis Sullivan ${ }^{1 *}$, Ethan Crowell', Sean Mossman ${ }^{2}$ and Mark G Kuzyk ${ }^{2}$

${ }^{1}$ Department of Electrical and Computer Engineering, University of Idaho, Moscow, Idaho, USA

${ }^{2}$ Department of Physics and Astronomy, Washington State University, Pullman, Washington, USA

\begin{abstract}
A method is described to simulate the magnetic susceptibility of a quantum toroid with grating. This simulation is based on the direct implementation of the time-dependent Schrödinger equation in three dimensions. The expectation value of the quantum magnetic dipole operator is calculated as a function of the applied magnetic field strength of a time-oscillating magnetic field. These expectation values are used to calculate the linear and nonlinear magnetic susceptibility of a torus.
\end{abstract}

\section{Keywords}

Nonlinear optics, Induced magnetic dipole, Quantum simulation, Magnetic Susceptibility

\section{Introduction}

Materials with nonlinear properties have applications in optical switching [1], lasers, photovoltaic cells [2], imaging [2], cancer therapy [3,4], advanced computing $[5,6]$, and communication technologies $[7,8]$. To improve applications, it is important to establish good methods and models to explore the optimization of nonlinear effects by increasing the nonlinear response. This work provides a way of calculating the nonlinear response of nanostructures. This helps in designing new systems without the need for doing difficult and time-consuming measurements.

The Finite-Difference Time-Domain (FDTD) method offers a method of directly implementing the Schrödinger equation in a three-dimensional structure and has recently been applied to quantum simulation [9-13]. The accuracy of this method in determining the eigenstates of quantum wires was previously described [14]. The FDTD method was used in the determination of the hyperpolarizability of quantum wires in close proximity to an electric dipole [15]. A method was also presented to simulate the magnetic response of a quantum toroid using the quantum magnetic dipole operator [16]. This work develops the FDTD method to calculate the magnetic dipole moment in a grated torus structure with a time-varying magnetic field. The magnetic susceptibilities are then calculated. This will allow for the optimization of structures to enhance the nonlinear properties in the presence of a time-varying magnetic field.

This paper will briefly describe the FDTD im-

*Corresponding author: Dennis Sullivan, Department of Electrical and Computer Engineering, University of Idaho, Moscow, Idaho, USA

Accepted: December 06, 2018; Published: December 08, 2018

Copyright: (C) 2018 Houle J, et al. This is an open-access article distributed under the terms of the Creative Commons Attribution License, which permits unrestricted use, distribution, and reproduction in any medium, provided the original author and source are credited.

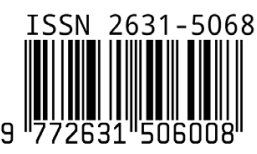

Houle J, et al. Int J Magnetics Electromagnetism 2018, 4:015 
plementation of the time-dependent Schrödinger equation and applies it to determine the eigenenergies and eigenstates of a quantum torus [17-19]. The FDTD implementation of the magnetic dipole moment operator will also be described [16]. Then a simulation will be presented for a time-harmonic electric field. Finally, the time-harmonic electric field will be used to calculate the magnetic susceptibility of a grated torus structure. The method presented may be used to calculate the magnetic susceptibility of other structures.

The Finite-Difference Time-Domain Method and the Determination of Eigenenergies and Eigenstates

The time-dependent Schrödinger equation [19] is given by:

$$
\begin{aligned}
\frac{\partial \Psi(x, y, z, t)}{\partial t}=i \frac{\hbar}{2 m_{\mathrm{e}}}[ & \left.\frac{\partial^{2} \Psi(x, y, z, t)}{\partial x^{2}}+\frac{\partial^{2} \Psi(x, y, z, t)}{\partial y^{2}}+\frac{\partial^{2} \Psi(x, y, z, t)}{\partial z^{2}}\right], \\
& -\frac{i}{\hbar} V(x, y, z) \Psi(x, y, z, t)
\end{aligned}
$$

Where $m_{e}$ is the mass of an electron and $V$ is the potential seen by the electron.

From [19], $\Psi$ contains both a real and an imaginary component. This allows $\Psi$ to be separated and Eq. (1) to be written as a pair of coupled equations which may be run sequentially:

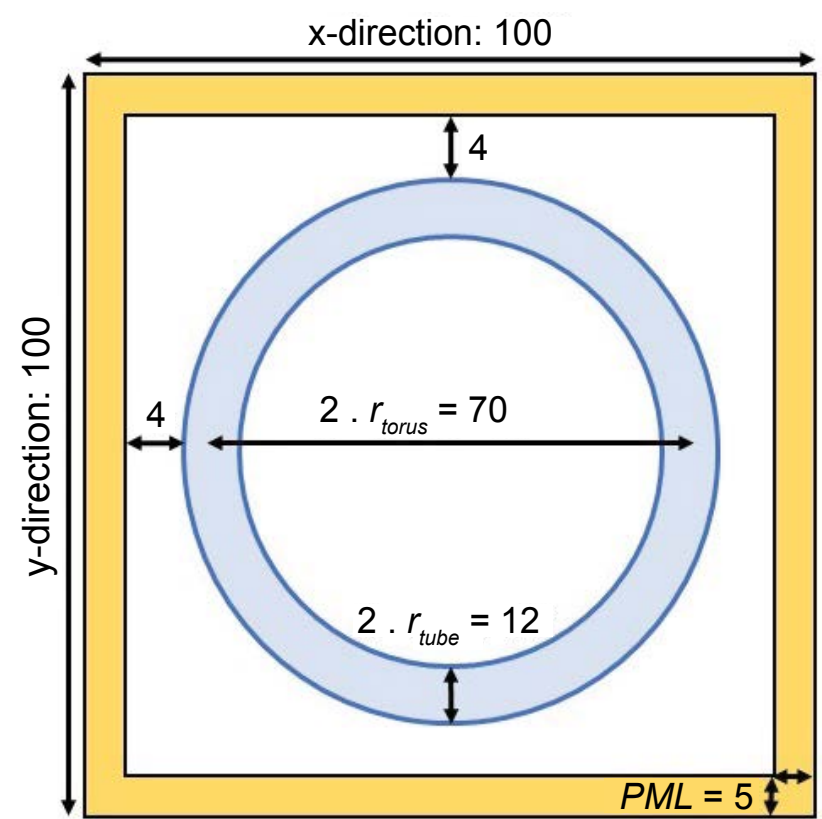

(a)

$$
\begin{aligned}
& \Psi_{\text {real }}^{k+1}(m, n, l)=\Psi_{\text {real }}^{k}(m, n, l)+\frac{\Delta t}{\hbar} V(m, n, l) \Psi_{\text {imag }}^{k+1 / 2}(m, n, l) \\
& -\frac{\hbar}{2 m_{e}} \frac{\Delta t}{(\Delta x)^{2}}\left[\begin{array}{l}
\Psi_{\text {imag }}^{k+1 / 2}(m+1, n, l)+\Psi_{\text {imag }}^{k+1 / 2}(m-1, n, l)+ \\
\Psi_{\text {imag }}^{k+1 / 2}(m, n+1, l)+\Psi_{\text {imag }}^{k+1 / 2}(m, n-1, l)+ \\
\Psi_{\text {imag }}^{k+1 / 2}(m, n, l+1)+\Psi_{\text {imag }}^{k+1 / 2}(m, n, l-1)-6 \Psi_{\text {imag }}^{k+1 / 2}(m, n, l)
\end{array}\right], \\
& \Psi_{\text {imag }}^{k+3 / 2}(m, n, l)=\Psi_{\text {imag }}^{k+1 / 2}(m, n, l)-\frac{\Delta t}{\hbar} V(m, n, l) \Psi_{\text {real }}^{k+1}(m, n, l) \\
& +\frac{\hbar}{2 m_{e}} \frac{\Delta t}{(\Delta x)^{2}}\left[\begin{array}{l}
\Psi_{\text {real }}^{k+1}(m+1, n, l)+\Psi_{\text {real }}^{k+1}(m-1, n, l)+ \\
\Psi_{\text {real }}^{k+1}(m, n+1, l)+\Psi_{\text {real }}^{k+1}(m, n-1, l)+ \\
\Psi_{\text {real }}^{k+1}(m, n, l+1)+\Psi_{\text {real }}^{k+1}(m, n, l-1)-6 \Psi_{\text {real }}^{k+1}(m, n, l)
\end{array}\right]
\end{aligned}
$$

The coordinates $m, n$, and I represent the physical positions in a matrix, which have replaced $x, y$, and $z$. Each cell has a linear dimension of $\Delta x$ on each side. Each time step iteration is represented by $k$, where the time $t$, from the beginning of the simulation, is given by $t=\Delta t \times k$ . These equations are evaluated for each cell in the three-dimensional array at each time step, and the alternating of the real and imaginary components allow the simulation of the behavior of $\Psi$ over time. Further details can be found in $[9-11,16,20]$. The $\Psi$ calculations over time can be used to find the eigenstates and eigenenergies [16-19].

This work will evaluate a torus with a diameter of 70 angstroms and a tube diameter rof 12 angstroms. The structure is created by setting a

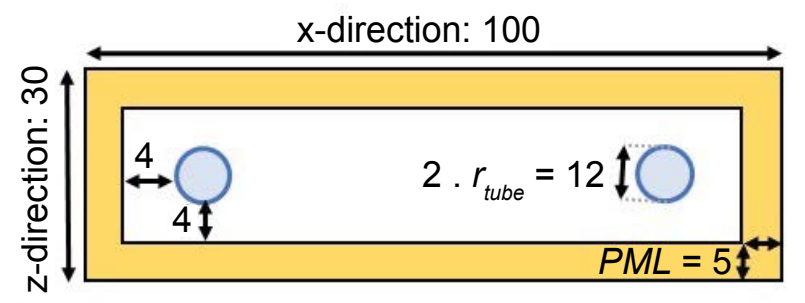

Figure 1: The problem space for the torus in the a) $x-y$ direction; and (b) $x-z$ direction. The total problem space is $100 \times 100 \times 30$ cells, with each cell representing one angstrom cubed. The torus radius is 35 angstroms, and the tube radius is 6 angstroms. The PML boundary is five cells in each direction. 
potential of $0 \mathrm{eV}$ for the cells composing the torus, and $4.6 \mathrm{eV}$ for the surrounding cells. A perfectly matched layer (PML) $[21,22]$ of five cells surrounds the torus to absorb outgoing waves as described in [13]. Figure 1 shows the simulation space for the torus.

In order to find the ground state eigenenergy, an initial test function is chosen for $\Psi\left(x, y, z, t_{0}\right)$. Details regarding the choice in test function were previously discussed [16]. The test function approximates the ground eigenstate by being evenly distributed around the torus with a Gaussian pulse according to

$$
\Psi\left(x, y, z, t_{0}\right)=\mathrm{e}^{-0.5 \cdot\left(\operatorname{dist}(x, y, z) /\left(0.5 r_{\text {tube }}\right)\right)^{2}},
$$

Where $\operatorname{dist}(x, y, z)$ is the distance from a given location to the center of the torus tube. The test function is shown in Figure 2.

The wavefunction is then monitored at the location $x_{0}, y_{0}, z_{0}$ over time, and a Fourier transform will allow the eigenenergy to be calculated, as shown in Figure 3. A single peak shows the ground state eigenenergy is $0.901 \mathrm{eV}$. This also shows the test function was a good estimation of the ground state, since no other peaks are visible to indicate other eigenenergies. Additional information can be found in [16].

Once the ground state eigenenergy is calculated, the ground eigenstate can be found by isolating it from the test function using the discrete Fourier transform at the frequency associated with the ground state eigenenergy, $\omega=\epsilon_{0} / \hbar$. The details of this process were previously described [16]. The resulting ground eigenstate is shown in Figure 4 through the center of the torus through the z-axis.

This result can be verified by calculating the frequency at which the ground eigenstate must oscillate. The period is defined by

$$
\mathrm{T}=\frac{\hbar}{\epsilon_{0}}=\frac{4.136 \times 10^{-15} \mathrm{eV} \cdot \mathrm{s}}{0.899 \mathrm{eV}}=4.6 \mathrm{fs} .
$$

To measure this period, the ground eigenstate is initialized to $\Psi_{\text {real }}$ and $\Psi_{\text {imag }}$. Then the FDTD program is run a designated number of iterations to ensure that the result is the expected behavior,

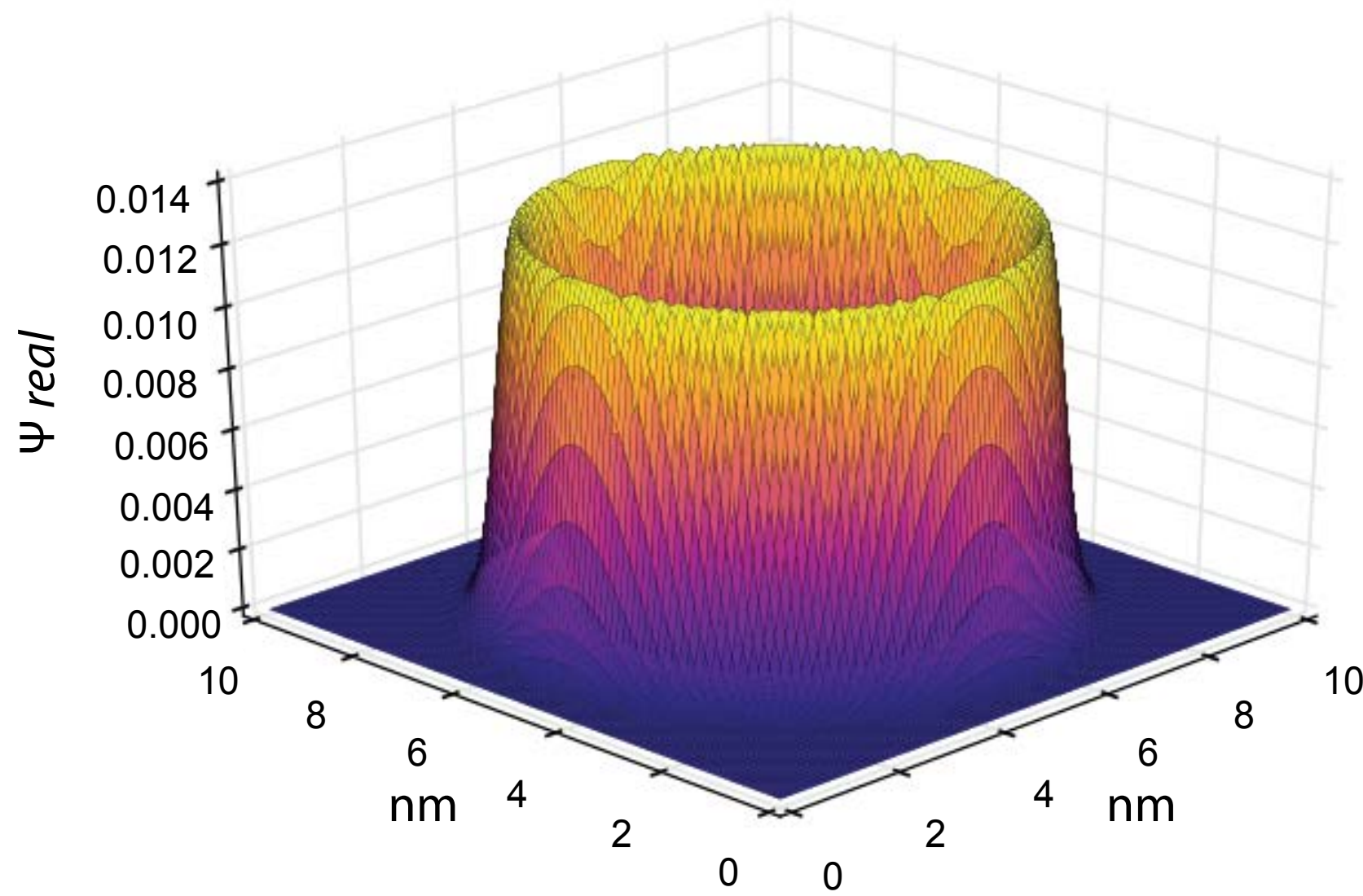

Figure 2: Test function initialized within a wire as the initial state $\Psi\left(x, y, z, t_{0}\right)$. This is a narrow Gaussian pulse centered within the torus. 


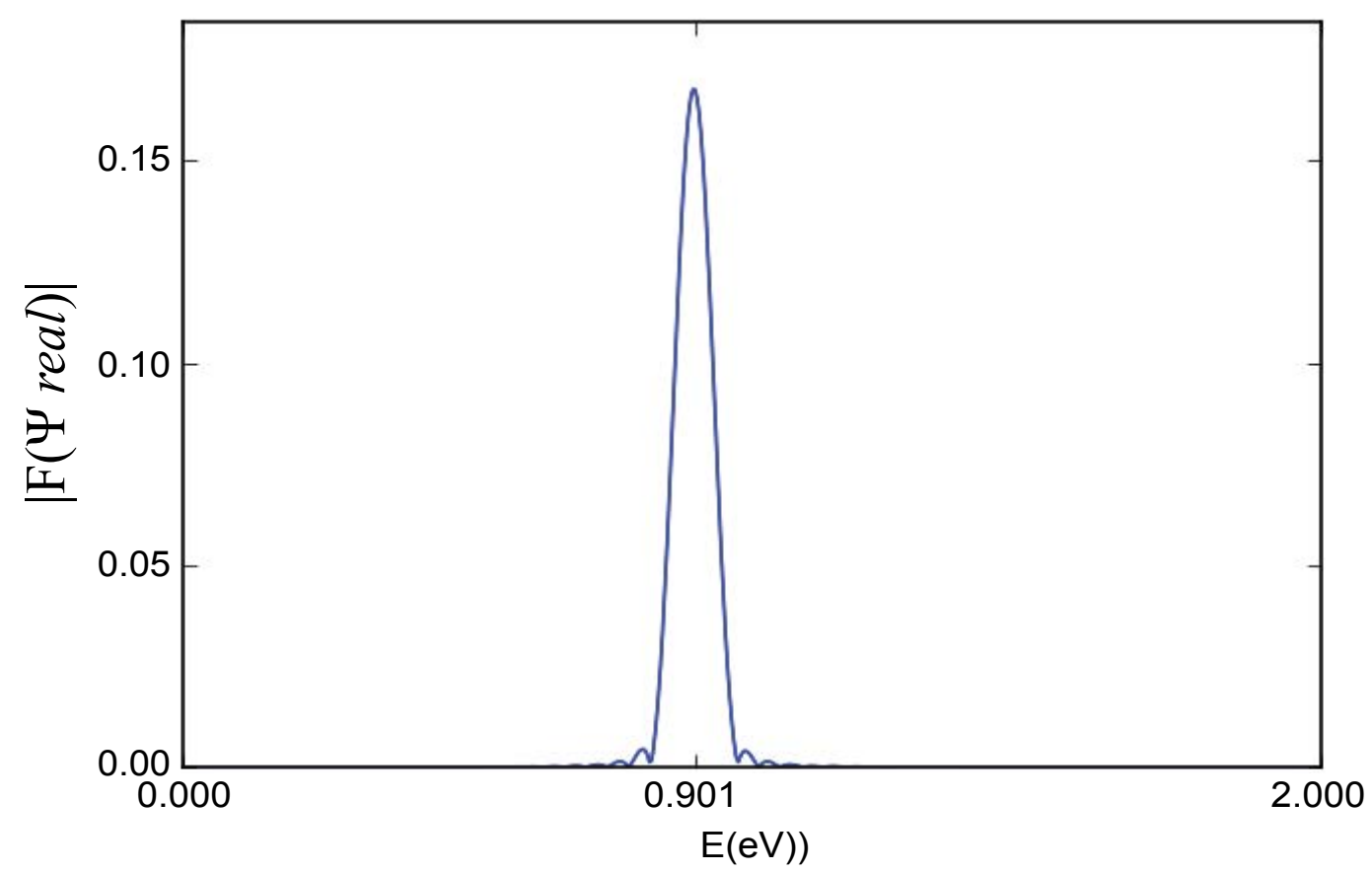

Figure 3: The eigenenergy distribution after 10,000 iterations beginning with a test function (shown in Figure 2) as the initial value for $\Psi\left(x, y, z, t_{0}\right)$. The resulting ground state eigenenergy is $0.901 \mathrm{eV}$.

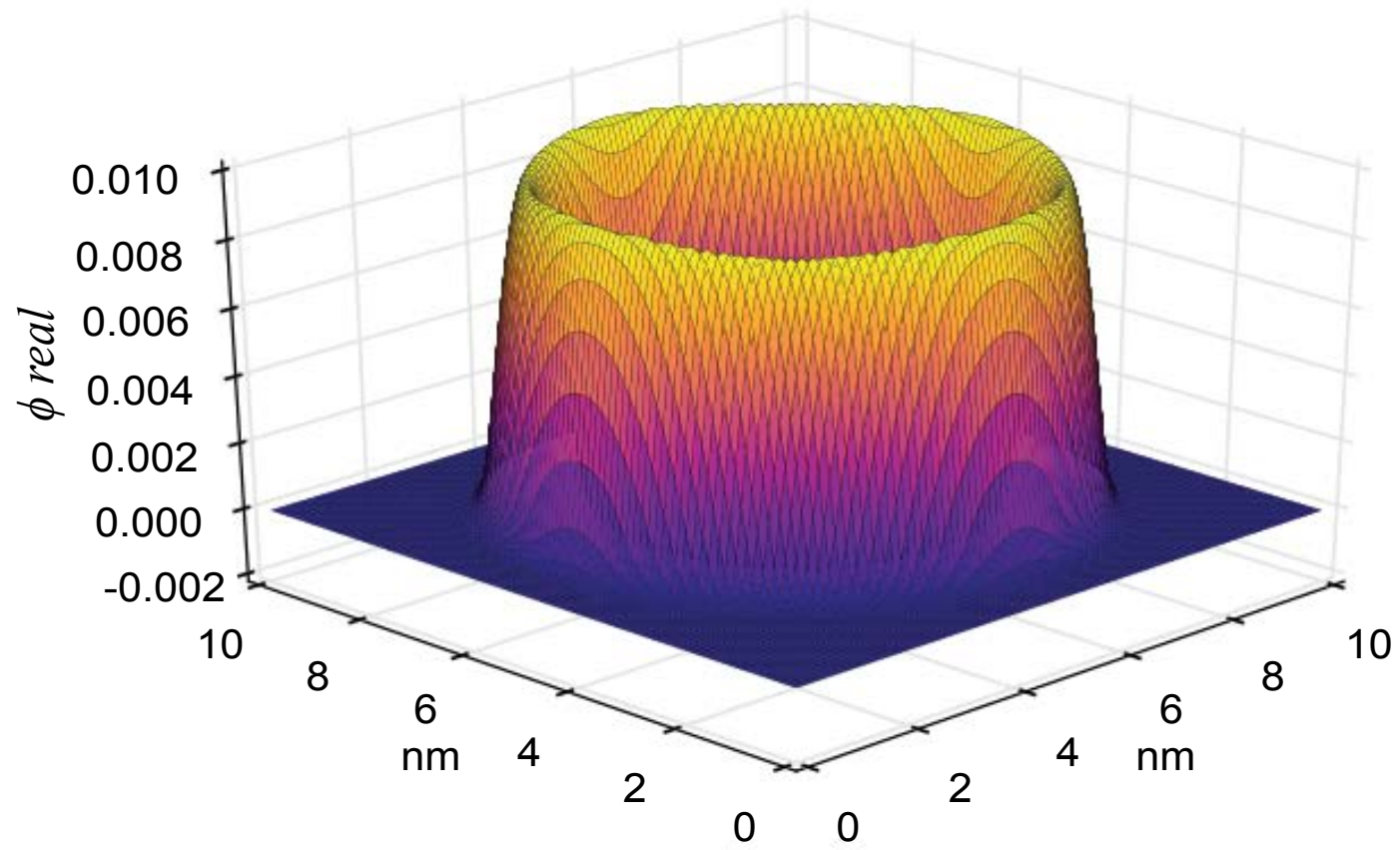

Figure 4: The ground eigenstate of the torus taken through the center of the torus. This was found using the discrete Fourier transform at the frequency associated with the ground state eigenenergy found in Figure 3.

as shown in Figure 5. A full period was shown to be 422-time steps, with the ground eigenstate inverted after 211-time steps.

\section{Calculation of the Magnetic Dipole Moment}

The Hamiltonian when a magnetic field is applied is given by

$$
H=\frac{1}{2 m_{\mathrm{e}}}\left(\frac{\hbar}{i} \nabla-q \cdot \mathbf{A}\right)^{2}+V(x, y, z)
$$

Where $\mathbf{A}$ is the vector potential [19]. A static magnetic field $B_{0}$ is applied in the z-direction, perpendicular to the torus. Then $\mathbf{A}$ can be simplified to 

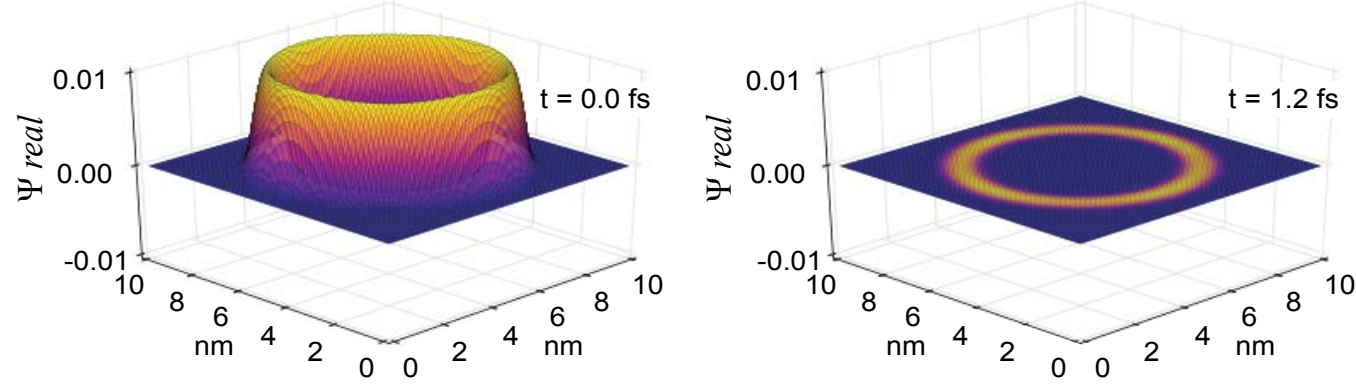

(a)

(b)
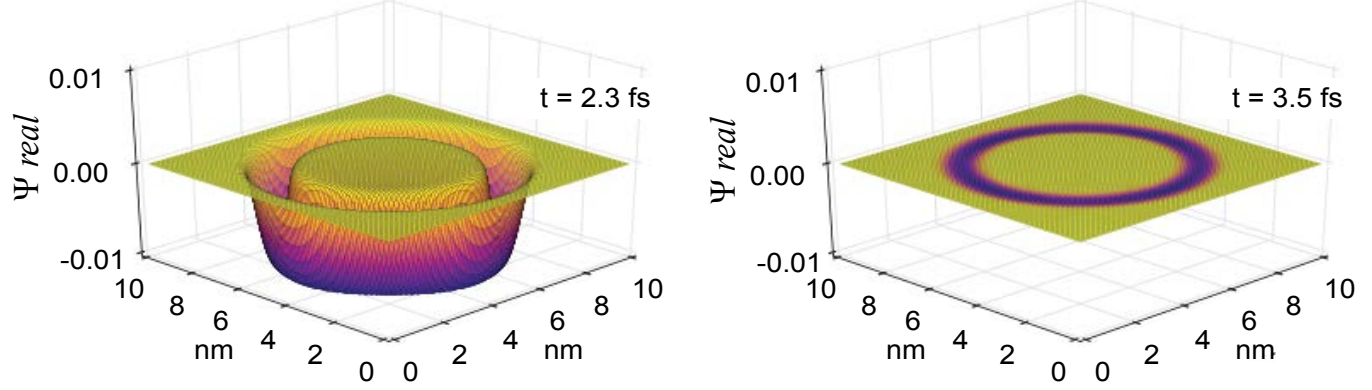

(c)

(d)

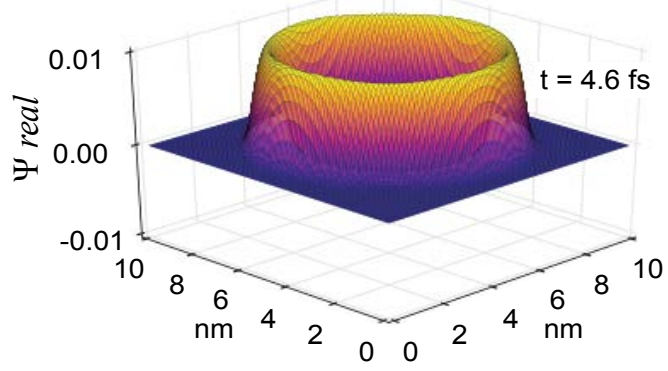

(e)

Figure 5: The ground eigenstate after a) 0 iterations (initial state); b) 106 iterations (quarter cycle); c) 211 iterations (half cycle); d) 317 iterations (three-quarters of a cycle), and e) 422 iterations (full cycle). The period of the ground state oscillating is $4.6 \mathrm{fs}$, which is the value predicted using the ground eigenenergy found in Figure 3.

$$
\mathbf{A}=\frac{1}{2}\left[-\left(y B_{0}\right) \hat{\mathbf{x}}+\left(x B_{0}\right) \hat{\mathbf{y}}\right]
$$

This results in the Hamiltonian:

$$
\begin{aligned}
H & =-\frac{\hbar^{2}}{2 m_{\mathrm{e}}}\left(\frac{\partial^{2}}{\partial x^{2}}+\frac{\partial^{2}}{\partial y^{2}}+\frac{\partial^{2}}{\partial z^{2}}\right)+i \frac{\hbar q B_{0}}{2 m_{\mathrm{e}}}\left(-y \frac{\partial}{\partial x}+x \frac{\partial}{\partial y}\right) \\
& +\frac{q^{2} B_{0}^{2}}{8 m_{\mathrm{e}}}\left(x^{2}+y^{2}\right)+V(x, y, z)
\end{aligned}
$$

This ignores the electric dipole interaction. Details of this implementation have been described previously $[9,10,16]$.

From [23], the magnetic dipole moment operator is

$$
\mathbf{m}=-\frac{1}{2} \frac{q}{m_{\mathrm{e}}} \frac{\hbar}{i}(\mathbf{r} \times \nabla)-\frac{q^{2}}{4 m_{\mathrm{e}}} B_{\mathrm{z}}(t) \cdot\left(x^{2}+y^{2}\right) .
$$

This is then implemented in FDTD, with the $x$ and $y$ positions being taken from the center of the torus. A detailed explanation of the FDTD implementation was previously given [16].

The accuracy of the expectation value of the magnetic dipole moment can be verified by comparing it with the classical magnetic dipole moment given by

$$
m=I \cdot \pi \cdot r_{\text {torus }}^{2} \hat{z},
$$


Table 1: Classical and quantum magnetic dipole moment with a static magnetic field.

\begin{tabular}{|l|l|l|l|}
\hline Magnetic Field $[\mathbf{T}]$ & Classical $\mathbf{m}\left[\mathrm{mA} \cdot(\AA)^{2}\right]$ & Operator $\mathbf{m}\left[\mathrm{mA} \cdot(\AA)^{2}\right]$ & Difference \\
\hline-50 & 7.8 & 7.6 & $2.6 \%$ \\
\hline-25 & 7.5 & 7.4 & $1.3 \%$ \\
\hline 0 & 7.3 & 7.2 & $1.4 \%$ \\
\hline 25 & 7.1 & 7.0 & $1.4 \%$ \\
\hline 50 & 6.9 & 6.8 & $1.5 \%$ \\
\hline
\end{tabular}

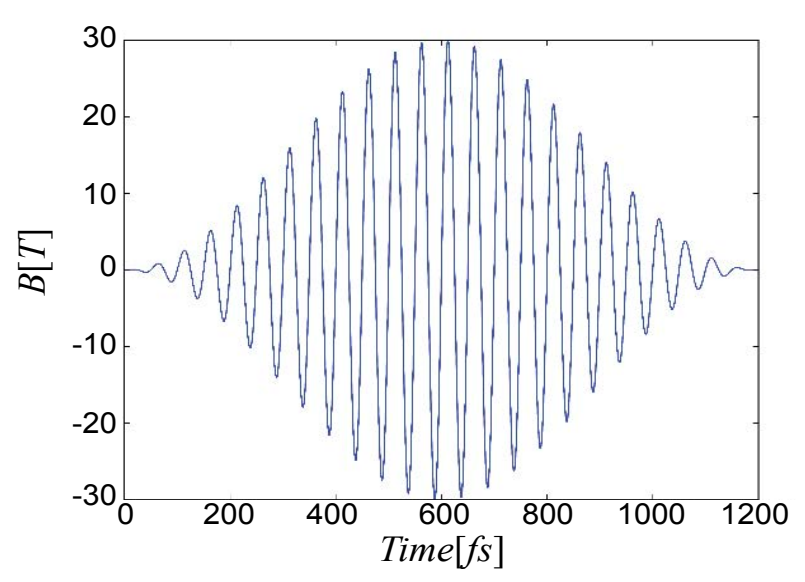

(a)

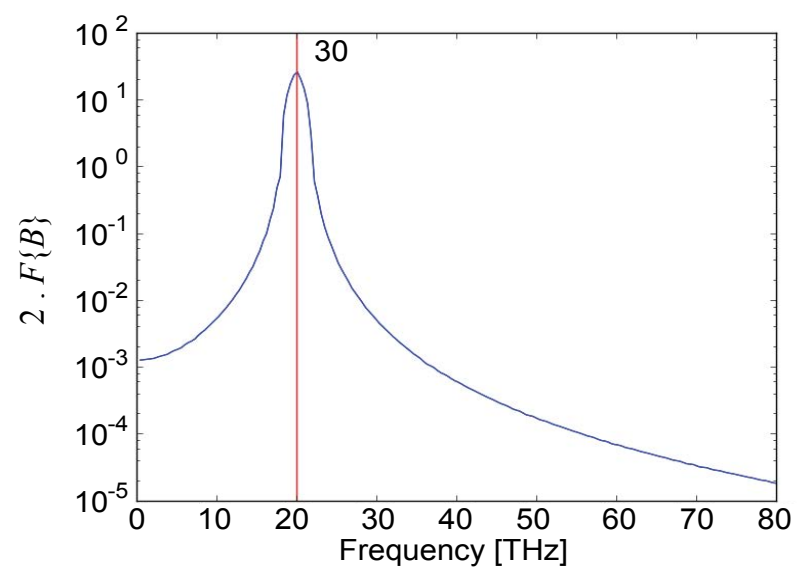

(c)

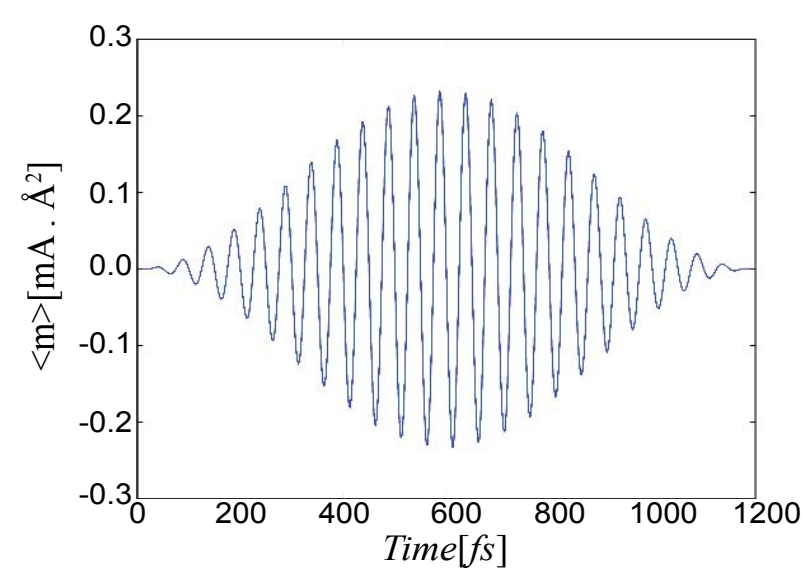

(b)

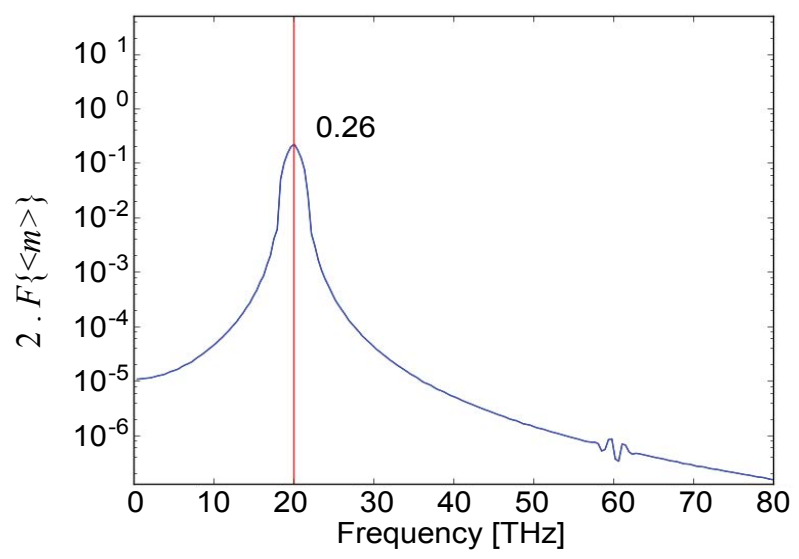

(d)

Figure 6: The results of a simulation of a particle in a torus under the influence of a sinusoidal magnetic field with a maximum magnitude of $30 \mathrm{~T}$ and a frequency of $20 \mathrm{THz}$. a) The applied magnetic field over time; b) The calculated magnetic dipole moment over time; c) The Fourier transform of the magnetic field showing the applied magnetic field strength of $30 \mathrm{~T}$ at $20 \mathrm{THz}$, and d) The Fourier transform of the dipole moment showing the primary response is at the applied frequency of $20 \mathrm{THz}$.

Where $l$ is the current and $r_{\text {torus }}$ is the radius of the torus [24]. A wave-packet within a radial Gaussian envelope is initialized in the torus and assigned the charge of one electron. The wave-packet will travel around the torus as the FDTD simulation runs. The expectation value of the position is used to determine the current in the torus. Further details regarding this method were previously described [16].
Table 1 shows the results of the classical and FDTD quantum approach for several magnetic field strengths. The classical and quantum approaches give values that are the same within about three percent, verifying the magnetic dipole moment operator and the magnetic field implementation using the FDTD method. Similar results were previously described, including a comparison at various eigenstates [16]. 
Simulation of a Time-Harmonic Magnetic Field

With the magnetic dipole moment operator verified, the effects of a time-harmonic magnetic field on the ground eigenstate can be examined. The ground eigenstate is loaded for the initial $\Psi_{\text {real }}$ and $\Psi_{\text {imag }}$. The applied magnetic field is a sinusoidal function,

$$
B(t)=B_{\max } \cdot \sin \left(2 \pi f_{0} t\right) \hat{z} .
$$

This will be multiplied by a Hanning window to reduce the effects of the abrupt addition of a magnetic field. The effects of the magnetic field may be monitored by observing the magnetic dipole moment using the operator previously described.

Figure 6a shows the applied $30 \mathrm{~T}, 20 \mathrm{THz}$ magnetic field over time. Figure $6 \mathrm{~b}$ shows the calculated magnetic dipole moment over time. Figure $6 \mathrm{c}$ and Figure $6 \mathrm{~d}$ show the Fourier transforms of the magnetic field and the magnetic dipole moment, respectively. The peak for the magnetic field is 30 Tat $20 \mathrm{THz}$ in Figure $6 \mathrm{c}$, as expected given the input magnetic field. The magnetic dipole moment shown in Figure $6 \mathrm{~d}$ also shows a peak at $20 \mathrm{THz}$ and no significant response at other frequencies.

The peak values of the magnetic dipole moment generated with the Fourier transform are determined at $f_{0}$. These are shown to have the expected linear relationship with the applied magnetic field strength, as shown in Figure 7.

\section{Magnetic Susceptibility}

The magnetic dipole moment generated from the FDTD method using the Fourier transform, as in Figure $6 \mathrm{~d}$, can be used to find the magnetic susceptibility.

From [1], the bulk polarizability is given by $P(t)=\chi^{(1)} E(t)+\chi^{(2)} E^{2}(t)+\chi^{(3)} E^{3}(t)+\ldots \ldots$,

Where $\chi^{(1)}$ is the linear electric susceptibility, $\chi^{(2)}$ is the second-order nonlinear optical susceptibility, and $\chi^{(3)}$ is the third-order nonlinear optical susceptibility. This response is in the z-direction only, which is the direction of the applied magnetic field. The bulk polarizability is related to the dipole moment of a molecule by

$$
P(t)=N p(t) \text {, }
$$

Where $N$ is the number density and many conditions are met [1]. The dipole moment equation may then be written as

$$
p(t)=\alpha E(t)+\beta E^{2}(t)+\gamma E^{3}(t)+\ldots \ldots .
$$

An analogous equation for the magnetic dipole moment $m$ using the magnetic field $B(t)$ results in

$$
m(t)=\alpha^{\prime} B(t)+\beta^{\prime} B^{2}(t)+\gamma^{\prime} B^{3}(t)+\ldots,
$$

Where the primed quantities are the linear and

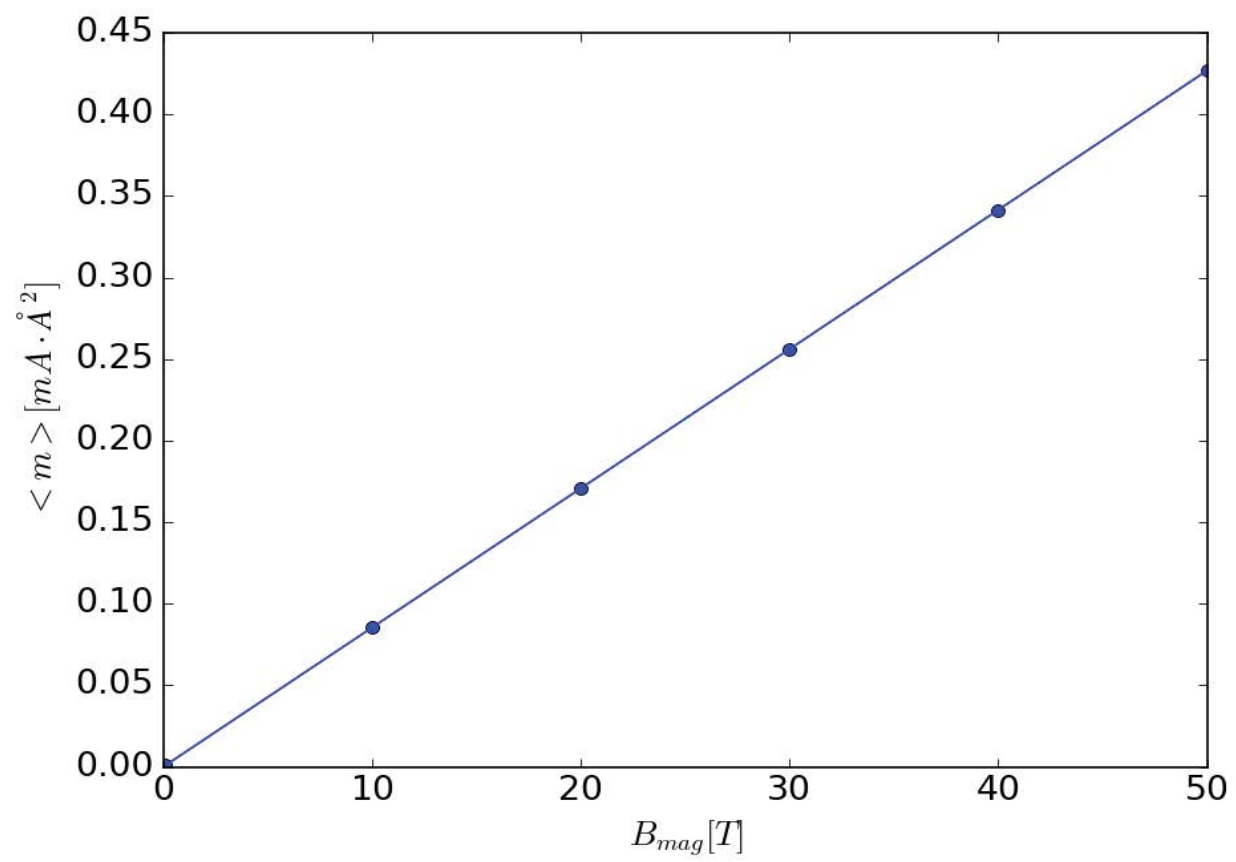

Figure 7: The magnetic dipole moment versus the magnetic field strength for a magnetic field of $20 \mathrm{THz}$. This shows the expected linear response. 


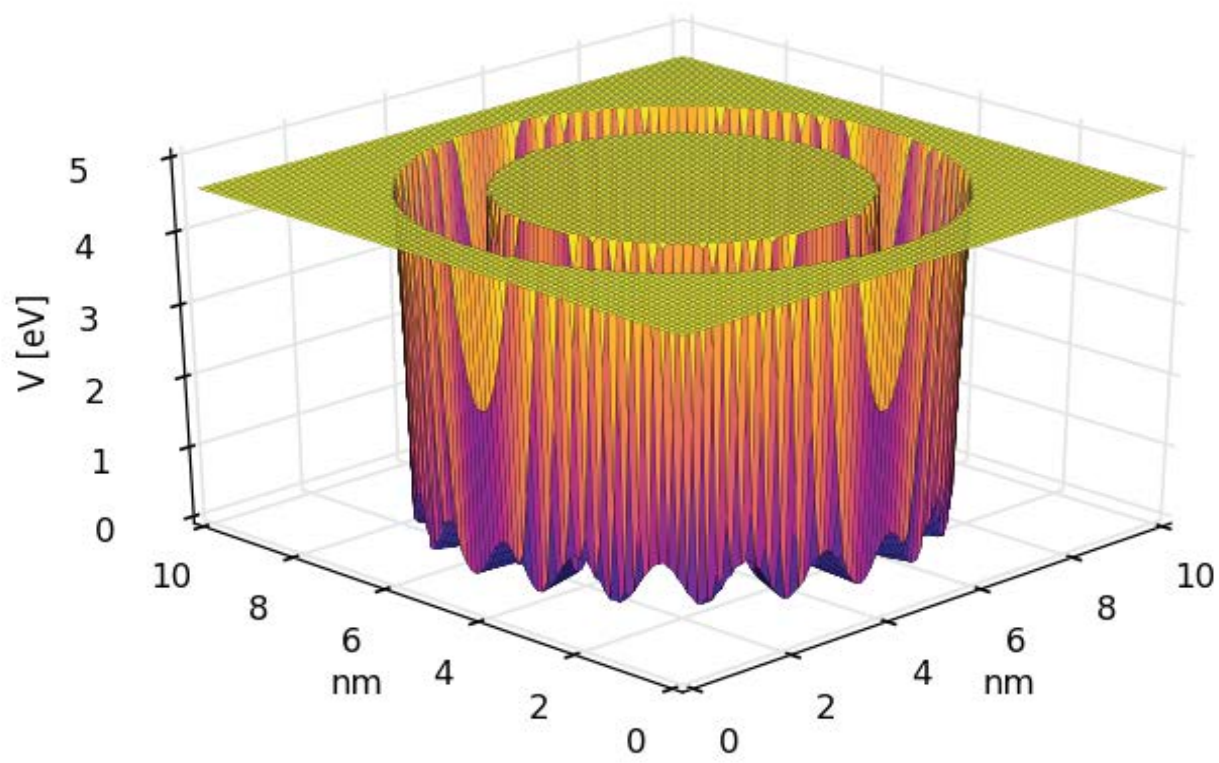

Figure 8: The potential showing a grated torus with the cross section at $z=Z_{c}$. This structure is used to find a nonlinear response.

nonlinear electric polarizabilities and hyperpolarizabilities. The following calculations will focus on $\alpha$ (the magnetic polarizability), $\beta$ (the magnetic hyperpolarizability), and $\gamma$ (the magnetic second hyperpolarizability) [2]. The response is needed in the frequency domain, so the magnetic dipole moment is written as

$$
m(\omega)=\alpha^{\prime} B(\omega)+\beta^{\prime} B^{2}(\omega)+\gamma^{\prime} B^{3}(\omega) .
$$

A Fourier transform is performed to obtain the magnetic dipole moment in the frequency domain.

The polarizability will occur at the fundamental frequency $\omega_{0}$. The time varying magnetic field was previously described in Eq. (9). This means the hyperpolarizability will have a magnetic field component

$$
B^{2}(t)=\frac{1}{2}\left(B_{\max }^{2}-B_{\text {max }}^{2} \cos \left(2 \omega_{0} t\right)\right),
$$

Which has a frequency component at 0 and at $2 \omega_{0}$ [25].

Similarly, the second hyperpolarizability will have a magnetic component

$$
B^{3}(t)=\frac{1}{4}\left(3 B_{\max }^{3} \sin \left(\omega_{0} t\right)-B_{\max }^{3} \sin \left(3 \omega_{0} t\right)\right)
$$

This means the second hyperpolarizability will have frequency components at $\omega_{0}$ and $3 \omega_{0}$.

The Fourier transform of the simulation results for the plain torus in Figure $6 \mathrm{~d}$ showed only a small component at $3 f_{0}$. In an attempt to find a higher nonlinear response, irregularities were added to the structure. A grated torus, one in which a periodic potential is added, is used in this work. Any structure may be examined using the FDTD method simply by specifying the potential $V(x, y, z)$.

The grated torus is shown in Figure 8. The torus shown has 20 peaks, and the difference between the peaks and the troughs is $0.6 \mathrm{eV}$. The corresponding ground eigenstate is shown in Figure 9 and also displays 20 peaks.

The results of a magnetic field described by Eq. (9) where $B_{\max }=30 \mathrm{~T}$ and $f_{0}=20 \mathrm{THz}$ on the grated torus are shown in Figure 10. The majority of the signal for the magnetic dipole moment is at $f_{0}$, but there is also a significant component at $3 f_{0}$ , as shown in Figure 10d. This corresponds with the second hyperpolarizability. This component is much more prominent than that seen in the plain torus in Figure $6 \mathrm{~d}$.

Figure 11 shows the component of the magnetic dipole moment determined at $20 \mathrm{THz} f_{0}$, across several magnetic field strengths. Because the hyperpolarizability has a cubic component at $f_{0}$, as shown in Eq. (16), a best fit line is created using a linear and a cubic response to match the calculated values. The linear coefficient, $\alpha$, is dominant and gives the polarizability.The cubic coefficient, $\gamma$, is a component of the second hyperpolarizability. These coefficients are used to create the solid fit line on the graph. 


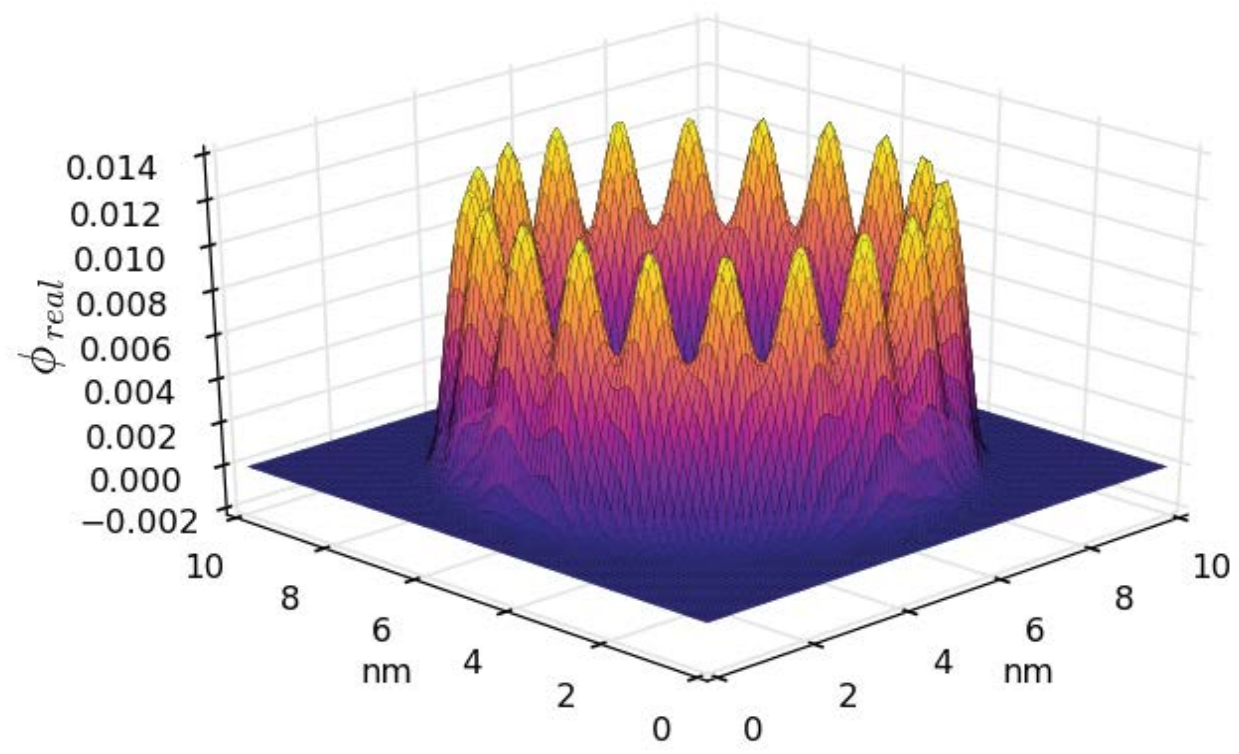

Figure 9: The ground eigenstate for the grated potential with the cross section shown at $z=Z_{c}$. This ground eigenstate will be used for the initial value $\Psi\left(x, y, z, t_{0}\right)$ when applying the time harmonic magnetic field.

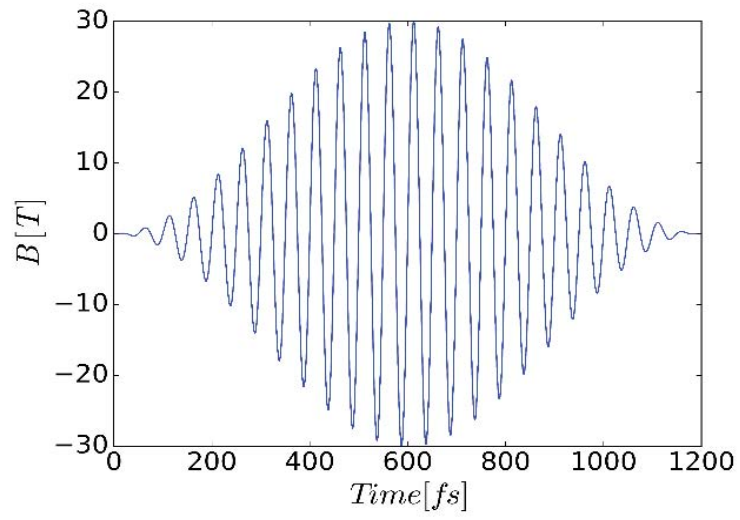

(a)

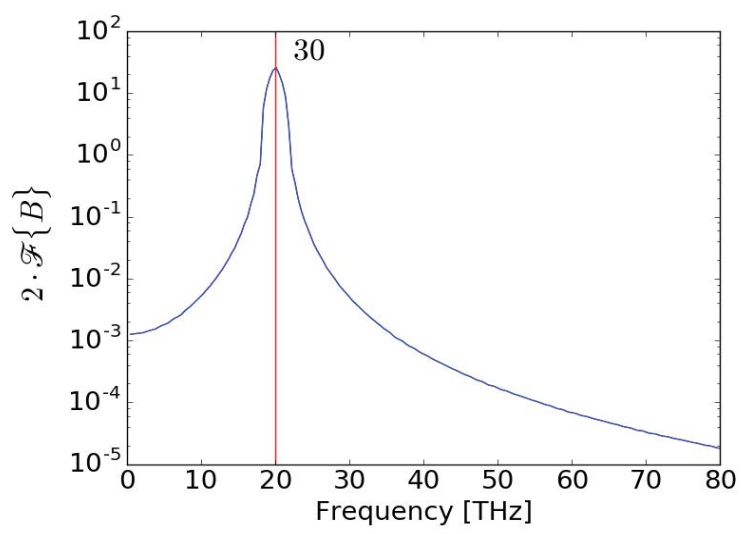

(c)

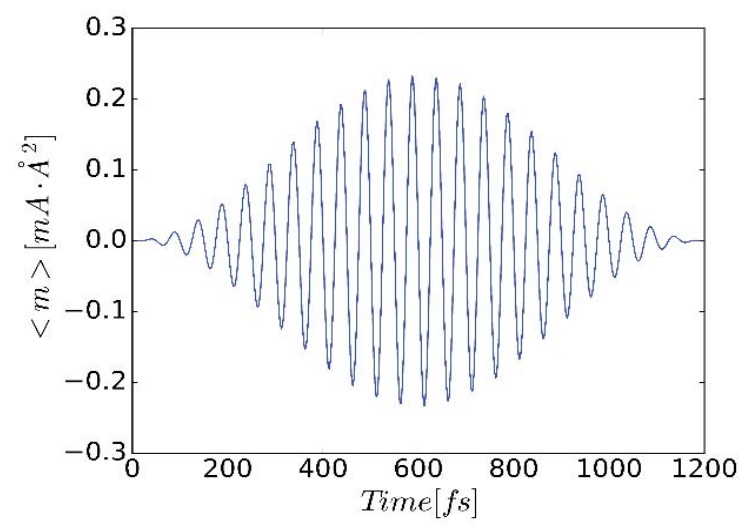

(b)

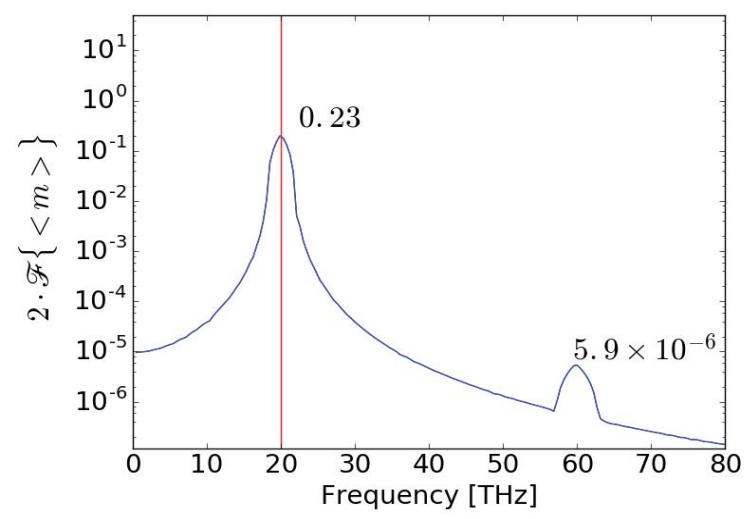

(d)

Figure 10: The results of a simulation of a particle in a grated torus under the influence of a sinusoidal magnetic field with a maximum magnitude of $30 \mathrm{~T}$ and a frequency of $20 \mathrm{THz}$. a) The applied magnetic field over time; b) The calculated magnetic dipole moment over time; c) The Fourier transform of the magnetic field showing the applied magnetic field strength of $30 \mathrm{~T}$ at $20 \mathrm{THz}$; and d) The Fourier transform of the dipole moment showing the primary response is at the applied frequency of $20 \mathrm{THz}$ and a secondary response at $60 \mathrm{THz}$. 


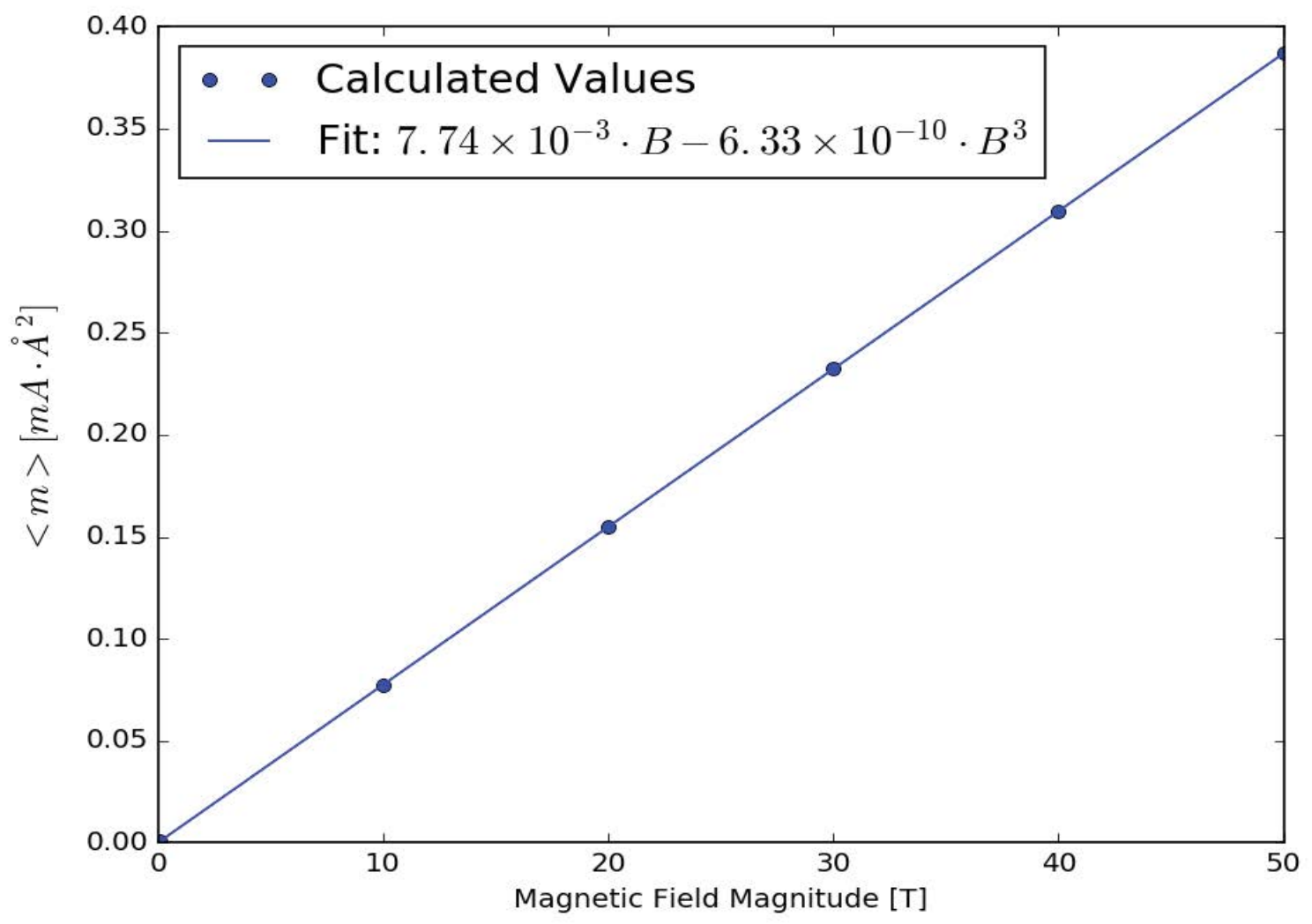

Figure 11: The absolute value of the magnetic dipole moment determined at $f_{0}$ versus magnetic field strength for a particle in a grated torus. A best fit line is used to find the linear coefficient $\left(\alpha=7.74 \times 10^{-3} \mathrm{~mA} \cdot \AA^{2} \cdot \mathrm{T}^{-1}\right)$ and the cubic coefficient $\left(\gamma=-6.33 \times 10^{-10} \mathrm{~mA} \cdot \AA^{2} \cdot \mathrm{T}^{-3}\right)$.

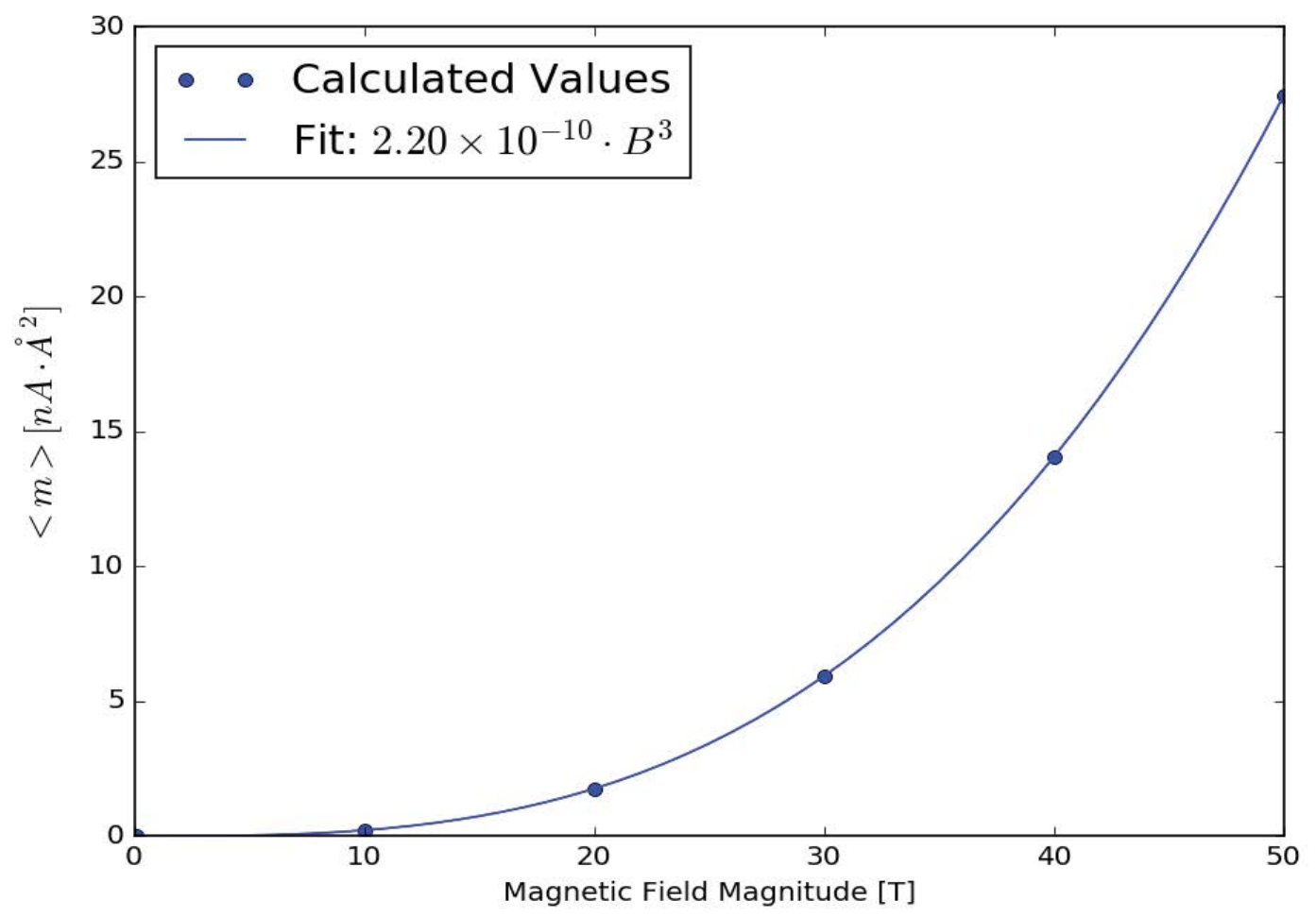

Figure 12: The absolute value of the magnetic dipole moment determined at $3 f_{0}$ versus magnetic field strength. A best fit line is used to find the cubic coefficient $\left(\gamma=2.20 \times 10^{-10} \mathrm{~mA} \cdot \AA^{2} \cdot T^{-3}\right)$. 
Similarly, the magnetic dipole moment from the Fourier transform can be determined at $2 f_{0}$, which is the frequency of the second harmonic, to examine the hyperpolarizability. In the case of both the plain and grated torus, this component is not present in any significant amount.

Figure 12 shows the component of the magnetic dipole moment at $3 f_{0}$. The coefficient of the cubic response is a component of $\gamma$, the second hyperpolarizability. The cubic fit line is also shown in Figure 11. The ratio of $\gamma$ determined at $3 f_{0}$ to $\gamma$ determined at $f_{0}$ is approximately -0.35 , which is close to the ideal value of $-1 / 3$. This ratio can be seen in Eq. (16).

\section{Conclusion}

A method was introduced that uses the quantum FDTD calculation to determine the magnetic susceptibility of a torus and of a grated torus. The expectation value of the magnetic dipole moment was found as a function of the applied time-varying magnetic field amplitude to determine the magnetic susceptibility. The response of a torus was found to be linear, with no significant frequency components aside from that of the applied frequency. For the grated torus, the magnetic dipole moment at the third harmonic displays a cubic response to magnetic field strength, as expected. The ratio of $\gamma$ determined at $3 f_{0}$ to $\gamma$ determined at $f_{0}$ was close to the predicted ratio of $-1 / 3$.

These simulations were done on a laptop computer with a $2.2 \mathrm{GHz}$ processor and $8 \mathrm{~GB}$ of RAM. A time-varying simulation at a single frequency, as shown in Figure 6, took approximately 10 minutes.

The FDTD method can simulate any structure, subject only to the resolution of the cell size and the dimensions of the problem space. This allows different nanostructures to be simulated, tested, and modified to search for the optimal nonlinear optical response. Future work will focus on creating these optimized structures.

\section{Acknowledgements}

$E C, S M$, and MGK acknowledge the generous support of the National Science Foundation, Grant ECCS-1128076.

\section{References}

1. RW Boyd (2003) Nonlinear optics. (2 ${ }^{\text {nd }}$ edn), Academic Press.
2. J Pérez-Moreno, S Shafei, MG Kuzyk (2013) Sum rules and scaling in nonlinear optics. Physics Reports 524: 397-398.

3. M Firczuk, M Winiarska, A Szokalska, M Jodlowska, M Swiech, et al. (2011) Approaches to improve photodynamic therapy of cancer. Front Biosci (Landmark Ed) 16: 208-224.

4. I Roy, TY Ohulchanskyy, HE Pudavar, EJ Bergey, AR Oseroff, et al. (2003) Ceramic-based nanoparticles entrapping water-insoluble photosensitizing anticancer drugs: A novel drug-carrier system for photodynamic therapy. J Am Chem Soc 125: 7860-7865.

5. DJ Brod, J Combe (2016) Passive CPHASE gate via cross-Kerr nonlinearities. Phys Rev Lett 117: 080502.

6. DJ Brod, J Combes, J Gea-Banacloche (2016) Two photons co- and counter propagating through $N$ cross-Kerr sites. Phys Rev A 94: 023833.

7. D Cotter, RJ Manning, KJ Blow, AD Ellis, AE Kelly, et al. (1999) Nonlinear optics for high-speed digital information processing. Science 286: 1523-1528.

8. JE Heebner, RW Boyd (1999) Enhanced all-optical switching by use of a nonlinear fiber ring resonator. Opt Lett 24: 847-849.

9. DM Sullivan, DS Citrin (2001) Time-domain simulation of two electrons in a quantum dot. Journal of Applied Physics 89: 3841-3846.

10. DM Sullivan, DS Citrin (2003) Time domain simulation of quantum spin. Journal of Applied Physics 94: 6518.

11. Soriano, EA Navarro, PA Porti, V Such (2004) Analysis of the finite difference time domain technique to solve the Schrödinger equation for quantum devices. Journal of Applied Physics 95: 8011-8018.

12. GB Ren, JM Rorison (2004) Eigenvalue problem of the Schrödinger equation via the finite-difference time-domain method. Phys Rev E Stat Nonlin Soft Matter Phys 69: 036705.

13. DM Sullivan, PM Wilson (2012) Time-domain determination of transmission in quantum nanostructures. Journal of Applied Physics 121: 064325.

14. DM Sullivan, S Mossman, M Kuzyk (2016) Timedomain simulation of three-dimensional quantum wires. PLoS One 11: e0153802.

15. DM Sullivan, S Mossman, M Kuzyk (2016) Hybrid quantum systems for enhanced nonlinear optical susceptibilities. Journal of the Optical Society of America B 33: E143-E149.

16. J Houle, DM Sullivan, E Crowell, S Mossman, MG Kuzyk 
(2018) Three-dimensional time domain simulation of the quantum magnetic dipole. International Journal of Magnetics and Electromagnetism 4: 011.

17. DM Sullivan, DS Citrin (2002) Determination of the eigenfunctions of arbitrary nanostructures using time domain simulation. Journal of Applied Physics 91: 3219-3226.

18. DM Sullivan, DS Citrin (2005) Determining quantum eigenfunctions in three-dimensional nanoscale structures. Journal of Applied Physics 97: 104305.

19. DM Sullivan (2012) Quantum mechanics for electrical engineers. IEEE Press.

20. W Dai, G Li, R Nassar, S Su (2005) On the stability of the FDTD method for solving a time-dependent Schrödinger Equation. Numerical Methods for
Partial Differential Equations 21: 1140-1154.

21. JP Berenger (1994) A perfectly matched layer for the absorption of electromagnetic waves. Journal of Computational Physics 114: 185-200.

22. C Zheng (2007) A perfectly matched layer approach to the nonlinear Schrödinger wave equations. Journal of Computational Physics 227: 537-556.

23. AL Fetter, JD Walecka (2003) Theoretical mechanics of particles and continua. Dover Publishing, New York.

24. DK Cheng (1989) Field and wave electromagnetics. Addison-Wesley Publishing.

25. MG Kuzyk (2017) Nonlinear optics: A Student's Perspective with python problems and examples. Createspace. 